\title{
Amours cachées : la nouvelle rhétorique mondaine bretonne
}

Hidden love: the new Breton rhetoric of the fashionable set

\section{Ronan Calvez}

\section{Q OpenEdition}

\section{Journals}

Édition électronique

URL : https://journals.openedition.org/lbl/1866

DOI : $10.4000 / \mathrm{lbl} .1866$

ISSN : 2727-9383

Éditeur

Université de Bretagne Occidentale - UBO

\section{Édition imprimée}

Date de publication : 1 février 2013

Pagination : 127-139

ISBN : 979-10-92331-00-4

ISSN : 1270-2412

\section{Référence électronique}

Ronan Calvez, «Amours cachées : la nouvelle rhétorique mondaine bretonne », La Bretagne

Linguistique [En ligne], 17 | 2013, mis en ligne le 01 mai 2021, consulté le 22 mai 2021. URL : http:// journals.openedition.org//bl/1866 ; DOl : https://doi.org/10.4000/lbl.1866

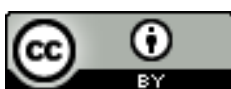

La Bretagne Linguistique est mise à disposition selon les termes de la Licence Creative Commons Attribution 4.0 International. 
Ronan CALVEZ*

\title{
Amours cachées : \\ la nouvelle rhétorique mondaine bretonne
}

«Belle, sans ornements, dans le simple appareil D'une beauté qu'on vient d'arracher au sommeil»

Racine, Britannicus (II, 2), 1669.

\begin{abstract}
D epuis que j'ai lu Kerenveyer et son Farvel göapaër $^{1}$, je crois être capable de reconnaître du breton mondain à la première lecture. Lorsque les chers collègues qui préparaient cette journée thématique sur la rhétorique m'ont demandé : «Et le breton mondain alors ?, j’ai accepté de me pencher sur la rhétorique mondaine pour voir si ce que j'avais inventé avait tout de même un sens et j'ai donc choisi d'analyser les trois sonnets du recueil de Kerenveyer. Pour deux raisons au moins : parce qu'ils forment un tout au sein de l'œuvre ; parce qu'ils relèvent d'un genre court bien représenté dans d'autres œuvres mondaines bretonnes et françaises.
\end{abstract}

Les voici, accompagnés d'une traduction.

* Professeur de celtique, CRBC (EA 4451), UBO/ueb

1. KERENVEYER, Ar farvel göapaër. Le bouffon moqueur, traduit et présenté par Ronan CALVEZ, CRBC - UBO, 2005. 


\section{Sonét la}

Va gouerc'h-'ted a viren abaouê pevar bloãs

Darniëgal, licaöui, ne rean eb quén,

Tost dar merc'hedigou, en hom dimillounén,

Evel-eur laoüénan, a zo voar ar meas.

Mæs cuittaât a ris va blijadur, sioüas !

Mac'harit a velis, ag e ris va c'houlén,

ar baötréz ne éllé neuze en hem divén,

rac en hem couroncat a réa en nöas ;

Tenna ris va c'huiban, ag e lâris dezi

Crock buan va velpen, ag en d'ha c'hours cus-'hi,

Frittomb prount carantes, eb quement a vez.

Ar foultret a rëa fæsoniou e leïs.

Tréus a ris gouscoude ar c'heff eus he greïs, göa ! petra gavis men ? an drouk eus a Naples.

Sonét. 2.

Étoués dousteriou eur, c'harantes tener

$E$ tremenén laouën, nemeur a nos vessou,

Va douçz a lavaré oán dezi quer

Va douçz a responte d'am oll santimanchou.

Mæs an derchen goall evel un anqueler,

en deus hi ravisset, d'am ampressamanc'hou

Siouas! ne joum din mui eus e zouster

nemit eur vras sourçzen a vestl ag a daëlou.

ah! me vouê trubuillet eus un taol quen terrubl

$E$ creis va zisesper e ris c'hriou orrubl,

Diblevet, divramet, dan oll é reañ truès.

Fantick d'ha zisparti a dichoät va c'halon...

Eur melconi quer bras a choueröa va buez

móc'h ambrouckin souden e sqaff ar gos Caron.

Sonét. 3.

$\mathrm{Vn}$ deis ho caquetal, gad certen fumellen e reañ va bossubl evit è discourna reud evel un ascorn oa va bitouzen c'hoas e vije cavet, me gred, ar calétta, Voär divron ar brincez un dorn a bourmenen un all a bresant ar felpen ar chaërra.

goa! nétra ne ellas na tomaat he stripen

ná tenna diout'hi ar guer an disterra.

Enfin disesperet e tennis va yalc'h, ar gast a lam voärni, en e dorn en dalc'h, ag prount en e vele, var guein en hem laqua, petiaoul, emesi, chom da darniegal ag epad peder heur en hem scuis ho meutta Laosqúit prount ar mounis, a roït vi diggaball. 


\section{Sonnet 1 er}

Depuis quatre ans, je conservais mon pucelage :

Je ne faisais que batifoler, cajoler,

Près des fillettes je me trémoussais

Comme un roitelet des campagnes.

Mais je quittai mon plaisir, hélas !

Je vis Mac'harit, et je fis ma demande.

La jeunesse ne pouvait alors se défendre,

Car elle se baignait nue.

Je sortis mon sifflet et je lui dis :

«Attrape vite ma grosse pièce, et dans ton con, cache-la,

Faisons promptement l'amour, sans plus de honte».

La fouteuse faisait quantité de manières.

Je traversai cependant sa souche du milieu.

Malheur! que trouvai-je ? le mal de Naples ${ }^{1}$.

\section{Sonnet 2}

Au milieu des douceurs d'un tendre amour Je passais joyeux bien des nuits.

Mon amante me disait que je lui étais cher,

Mon amante répondait à tous mes sentiments.

Mais la fièvre, mauvaise comme un génie,

L'a ravie à mes empressements.

Hélas ! il ne me reste de sa douceur

Qu'une grande source de fiel et de larmes.

Ah ! je fus affligé d'un coup si terrible,

Au milieu de mon désespoir, je poussai des cris effroyables,

Echevelé, dépenaillé, je faisais pitié à tous.

Fantick, ton départ fait saigner mon cœur.

Une mélancolie si grande rend ma vie tellement amère

Que je vous accompagnerai bientôt dans la barque du vieux Charon.

\section{Sonnet 3}

Un jour que je babillais avec certaine femelle, Je faisais mon possible pour la dégeler.

Mon vit était raide comme un os ;

On l'aurait trouvé, je crois, encore plus dur.

Sur les seins de la princesse, je promenais une main,

L'autre présente la superbe grosse pièce.

Malheur! rien ne put ni réchauffer son boyau,

Ni lui tirer le moindre mot.

Enfin, désespéré, je tirai ma bourse :

La putain saute dessus, l'empoigne,

Et promptement, dans son lit, elle se met sur le dos.

«A quoi bon, dit-elle, rester batifoler,

Et pendant quatre heures se fatiguer à patiner.

Lâchez promptement la monnaie, et donnez de l'œuf sans barguigner ». 
Il peut sembler bien audacieux de chercher à définir rhétoriquement des sonnets qui, à la première lecture, ne présentent, pour certains lecteurs, qu'un seul intérêt : enfin du cul en breton. Car il est vrai que ces sonnets comportent les premières occurrences écrites connues de mots désignant les attributs féminins et masculins ${ }^{2}$ : il y a donc un intérêt philologique évident. Mais ce n'est pas tout. En effet, dans des sonnets que d'aucuns feraient relever du genre poissard, Kerenveyer manie de nombreuses figures rhétoriques ainsi que des figures du savoir.

$*$

Ce paradoxe, comment l'entendre?

Dans les trois sonnets, on voit apparaître très clairement des figures rhétoriques, des figures de l'esprit.

\section{Sonét la}

Va gouerc'h-'ted a viren abaouê pevar bloãs

Darniëgal, licaöui, ne rean eb quén,

Tost dar merc'hedigou, en hom dimillounén,

Evel-eur laoüénan, a zo voar ar meas.

Mæs cuittaât a ris va blijadur, sioüas !

Mac'harit a velis, ag e ris va c'houlén,

ar baötréz ne éllé neuze en hem divén,

rac en hem couroncat a réa en nöas ;

Tenna ris va c'huiban, ag e lâris dezi

Crock buan va velpen, ag en d'ha c'hours cus-'hi, Frittomb prount carantes, eb quement a vez.

Ar foultret a rëa fæsoniou e leiss.

Tréus a ris gouscoude ar c'heff eus he greiss, göa ! petra gavis men ? an drouk eus a Naples.

\section{Sonét. 2.}

Étoués dousteriou eur, c'harantes tener $E$ tremenén laouën, nemeur a nos vessou, Va douçz a lavaré oán dezi quer Va douçz a responte d'am oll santimanchou.

Mæs an derchen goall evel un anqueler, en deus hi ravisset, d'am ampressamanc'hou Siouas ! ne joum din mui eus e zouster

métaphorisation française (darniëgal); accumulation (darniëgal + licaöui) hypocoristique (merc'hedigou) comparaison (evel eur laoüénan) exclamation (sioüas!)

métaphore (va c'huiban)

métaphore (va velpen) ; hypotypose (en

d'ha c'hours)

image (frittomb carantes)

métaphorisation française (ar foultret)

image (tréus ar c'heff eus he greïs)

exclamation (göa!) ; interrogation oratoire

(petra gavis men ?) ; image endormie (an drouk eus...)

image (dousteriou eur c'harantes)

anaphore (va douçz)

métaphorisation française (a responte d'am oll santimanchou)

comparaison (evel un anqueler)

métaphorisation fr. (hi ravisset d'am

ampressamanc'hou)

exclamation (siouas!)

2. Voir Martial MenARD, Alc'hwez bras ar baradoz vihan. Geriahudur ar Brezhoneg, An Here, 1995. 
nemit eur vras sourçzen a vestl ag a daëlou.

ah ! me vouê trubuillet eus un taol quen terrubl $E$ creis va zisesper e ris c'hriou orrubl, Diblevet, divramet, dan oll é reañ truès.

Fantick d'ha zisparti a dichoät va c'halon... Eur melconi quer bras a choueröa va buez móc'h ambrouckin souden e sqaff ar gos Caron. image (eur vras sourçzen)

exclamation (ah !) ; allégorie (un taol) métonymie (e creiz)

accumulation (diblevet, divramet) hypocoristique (Fantick) ; prosopopée (d'ha zisparti) ; image (a dichoät va c'halon) ; image (a choueröa va buez) prosopopée (moc'h ambrouckin) ; image (sqaff ar gos Caron)

\section{Sonét. 3.}

Vn deis ho caquetal, gad certen fumellen e reañ va bossubl evit è discourna reud evel un ascorn oa va bitouzen c'hoas e vije cavet, me gred, ar calétta, Voär divron ar brincez un dorn a bourmenen un all a bresant ar felpen ar chaërra. goa ! nétra ne ellas na tomaat he stripen ná tenna diout'hi ar guer an disterra. Enfin disesperet e tennis va yalc'h, ar gast a lam voärni, en e dorn en dalc'h, ag prount en e vele, var guein en hem laqua, petiaoul, emesi, chom da darniegal ag epad peder heur en hem scuis ho meutta Laosqúit prount ar mounis, a roït vi diggaball.

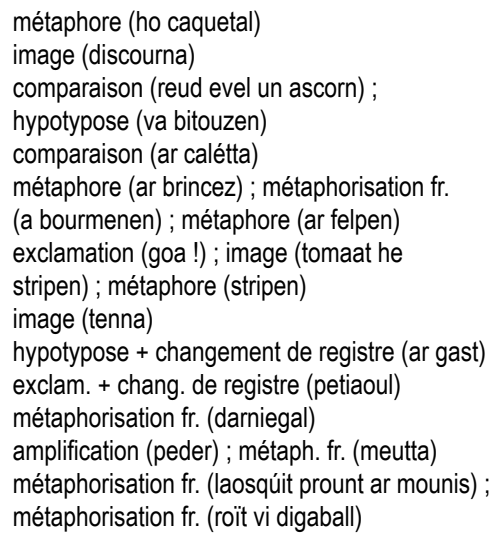

\section{Ces 42 vers comportent donc 52 figures que l'on peut classer} comme suit :

\begin{tabular}{|l|c|c|}
\hline Figures & Nombre & $\%$ \\
\hline Image & 11 & 26,19 \\
\hline Métaphorisation française & 9 & 21,42 \\
\hline Métaphore & 6 & 14,28 \\
\hline Exclamation & 6 & 14,28 \\
\hline Comparaison & 4 & 9,52 \\
\hline Hypotypose & 3 & 7,14 \\
\hline Accumulation & 2 & 4,76 \\
\hline Hypocoristique & 2 & 4,76 \\
\hline Prosopopée & 2 & 4,76 \\
\hline Changement de registre & 2 & 4,76 \\
\hline Allégorie & 1 & 2,38 \\
\hline Interrogation oratoire & 1 & 2,38 \\
\hline Anaphore & 1 & 2,38 \\
\hline Métonymie & 1 & 2,38 \\
\hline Amplification & 1 & 2,38 \\
\hline
\end{tabular}


J'ai dénombré 15 figures différentes. Néanmoins, les images et les métaphores sont parfois difficiles à distinguer : si on les assemble, elles représentent $40,47 \%$ des figures. Toutes deux, elles ont en commun de transporter brusquement l'esprit du lecteur - ou de l'auditeur - d'un objet à un autre : depuis l'Antiquité, la métaphore est considérée comme une preuve de la force d'invention ${ }^{3}$. Les comparaisons sont fondées sur le même procédé : les images, les métaphores et les comparaisons représentent 49,99\% - la moitié des figures. Vient ensuite la métaphorisation française qui est une des caractéristiques objectives distinguant les productions mondaines des contes, des chansons ou des bouts-rimés scatologiques en langue bretonne ${ }^{4}$ : certains mots bretons sont métaphorisés et passent d'un sens concret à un sens abstrait ${ }^{5}$. Ces mots bretons ne sont compréhensibles que par la connaissance que l'on doit avoir du champ sémantique de tel ou tel mot français ou de telle ou telle locution.

Le verbe «darniëgal» - qu'il faut lire sans doute «darnigeal» et que l'on trouve dans le premier sonnet -, signifie concrètement «voler bas». Ici, Kerenveyer élargit le champ sémantique du verbe, sur le modèle du français batifoler ${ }^{6}$. Dans ce même sonnet, le nom «foultret» ne se comprend que si on connaît la fouteuse ou la foutée, personnage récurrent de la littérature libertine ${ }^{7}$. Dans le deuxième sonnet, sans la connaissance des expressions françaises «répondre à des empressements ou à des sentiments » - dont de nombreuses occurrences existent dans la littérature classique française ${ }^{8}-$, on

3. Voir Marc-André BERNIER, Libertinage et figures du savoir. Rhétorique et roman libertin dans la France des Lumières (1734-1751), Les Presses de l'Université Laval, L'Harmattan, 2001, p. 82.

4. On en trouvera de nombreux exemples dans Émile ERNAULT, «Glossaire cryptologique du breton», Kryptadia, tomes II (1884), III (1886), VI (1899), VIII (1902).

5. Voir Ronan CALVEZ, «La métaphore mondaine. Kerenveyer et les littératures du breton », La Bretagne linguistique, volume 14, Brest, CRBC - UBO, 2009, p. 25-40.

6. On lit, dans le dictionnaire de Furetière : «batifoler. terme populaire, qui se dit de ceux qui s'amusent à se jouër, \& à badiner les uns avec les autres, particulièrement des paysans \& paysannes».

7. Voir, par exemple, SADE, La Nouvelle Justine ou les Malheurs de la vertu, suivie de l'Histoire de Juliette, sa sœur, 1797.

8. «Malgré l'empressement d'un curieux désir, / Il faut, pour lui parler, attendre son loisir» (Corneille, L'Illusion comique, I, 1, 1639) ; «Lorsqu'un homme vous 
n'entend rien à ce que dit l'auteur. Dans le troisième sonnet, le verbe «caquetal» renvoie très certainement au verbe français coqueter: «caresser, conter des fleurettes, dire des douceurs aux belles. C'est aussi faire des minauderies, \& affecter un air tendre, pour engager les femmes ${ }^{9} »$. Le verbe «meutta» est certainement construit à partir du nom meud 'pouce' ${ }^{10}$ et je le traduis par patiner : «Pour toucher, manier, tâter, farfouiller. [...] Dans le sens libre signifie aussi manier la nature d'une femme, la lui chatouiller des doigts, lui donner du plaisir. C'est ordinairement le prélude, pour passer plus outre ${ }^{11} \gg$. De même, le dernier vers est incompréhensible si l'on ne connaît pas le proverbe français cité par Furetière : «On dit aussi, que des petites gens donnent un $x u f$ pour avoir un bœuf, quand on fait de petits presents pour en attirer de plus gros. [...] On dit d'un avare, qu'il tondroit sur un $e u f$, qu'il ne donneroit pas un gros $e u f$ pour un petit.» De plus, il n'est pas interdit de penser que Kerenveyer joue ici sur l'homonymie entre le «vi» breton - qui signifie 'œuf' - et le vit français.

Ce que j'ai appelé la métaphorisation française et qui est opérée par Kerenveyer fonde, à l'évidence, l'identité littéraire de son œuvre : elle est la distance formelle qu'il introduit, en plus de la distance thématique induite nécessairement par la culture classique dont il émaille son œuvre. Ainsi, pour comprendre la chute du second sonnet, faut-il savoir que Charon est le nocher infernal de la mythologie grecque et romaine qui recevait les âmes des morts et leur faisait traverser l'Achéron au prix d'une obole.

Ces sonnets ne sont donc pas accessibles sans une connaissance $\mathrm{du}$ français et sans un solide bagage culturel. Kerenveyer fait preuve

vient embrasser avec joie, / Il faut bien le payer de la même monnoie, / Répondre, comme on peut, à ses empressements, / Et rendre offre pour offre, et serments pour serments» (Molière, Le Misanthrope, I, 1, 1666) ; «La jalousie est assez fine, / Et ces délicats sentiments / Méritent bien qu'on s'imagine / Que celui qui pour vous a ces empressements, / Passe le commun des amants » (Molière, Psyché, IV, 2, 1671).

9. Dictionnaire comique, satyrique, critique, burlesque, libre et proverbial... par Philibert-Joseph LE RouX, Amsterdam, 1750, première partie, p. 151.

10. François VALLÉE dans son Grand dictionnaire français-breton (Rennes, 19311933) donne meuda, meudata 'prendre par pincée, manier, compter la monnaie'.

11. Dictionnaire comique..., op. cit., deuxième partie, p. 173. 
d'une réelle éloquence - et il est intéressant de relever que ces traits, ces figures, se retrouvent dans d'autres textes bretons de la même période ${ }^{12}$ : il existe donc une éloquence mondaine bretonne qui dépasse le simple état de textes poissards. Le qualificatif qui me semble le mieux caractériser cette éloquence, c'est l'amplification : la lecture des sonnets, mais aussi des épigrammes ou des contes du même Kerenveyer, est à ce titre éloquente - c'est le cas de le dire. En effet, il use et abuse de figures, mais pas seulement comme ornementation baroque : il n'y a pas là que du cul, justement, et les figures de Kerenveyer ne sont pas là que pour faire joli - c'est donc aussi pour cela que notre auteur ne fait pas que du baroquisme.

À mon sens, il met en lumière une rhétorique bretonne mondaine. Et c'est une autre caractéristique de nos sonnets : ils font appel à des figures de savoir.

D’une certaine façon, Kerenveyer est passeur de mémoires : homme de son temps, il adapte aux lettres bretonnes la rhétorique du siècle des Lumières.

Cette rhétorique prend sa source principale dans l'enseignement développé par les jésuites ${ }^{13}$ et elle prend appui sur l'amplification. Cette dernière se trouve efficacement résumée par cet extrait d'un ouvrage de Dominique de Colonia qui, en 1710, dans son De arte rhetorica, écrit ${ }^{14}$ :

L'amplification permet d'affirmer avec plus de force, de manière à entraîner la persuasion par l'émotion qu'on suscite dans les esprits [...] A cette fin, on recourt surtout à six procédés : $1^{\circ}$ Les métaphores. $2^{\circ}$ Les hyperboles. $3^{\circ}$ Les synonymes. $4^{\circ}$ Les termes plus forts et plus éclatants. $5^{\circ}$ Les périphrases, ou circonlocutions. $6^{\circ}$ Les répétitions.

12. Voir Ronan CALVEZ, «Ange et bête. Au XVIII' siècle, en Basse-Bretagne : une sociabilité, mondaine et bretonne», dans Les enjeux thérapeutiques et esthétiques de la sociabilité au XVIII siècle, dirigé par Annick COSSIC-PÉRICARPIN et Hélène DACHEZ, Éditions Le Manuscrit, 2013.

13. Voir André Collinot, Francine MAZIÈre, L'exercice de la parole. Fragments d'une rhétorique jésuite, collection «Archives du commentaire», Éditions des Cendres, 1987.

14. Cité et traduit du latin par Marc-André BERnIER, op. cit., p. 108. 
Les objectifs pédagogiques de l'apprentissage de ces procédés sont très clairs et s'appuient sur le modèle cicéronien : instruire, plaire et toucher ${ }^{15}$. Ce qui semble être un programme peu différent de celui qui animait les auteurs de la littérature du breton des siècles précédents. En effet, si les textes religieux des XVI et XVII ${ }^{\mathrm{e}}$ siècles sont bel et bien les expressions littéraires formelles d'une posture catholique de sujétion aux règles divines de création du monde, il n'en est pas moins vrai que se plier à des règles de versification d'une très grande complexité et, dans ce cadre strict, user d'une licence de rythme et de rime ${ }^{16}$, est sans doute la mise en mots de la condition du chrétien : dans le cadre social imposé par l'Église, le chrétien peut user d'une liberté, contrôlée certes, mais bien sentie - ces textes sont donc aussi philosophiques et visent également à instruire, plaire et émouvoir. Néanmoins, au XVIII siècle, ces trois fonctions oratoires se doivent d'être mises en œuvre en associant le savoir à une figure, de façon systématique : l'une et l'autre doivent être liés. Dans ce cadre rhétorique, une pensée n'est réellement ingénieuse que lorsque la figure qui lui est liée laisse entendre - dans tous les sens du terme - le savoir ${ }^{17}$. Efficacement.

Sortie des collèges, la rhétorique paraît sur la «scène du monde» : du champ du sacré, elle glisse à celui du profane. Aussi, lorsqu'on lit les œuvres libertines françaises et les analyses rhétoriques qui ont été faites de ces dernières, ne peut-on manquer d'être frappé par les points communs entre cette rhétorique et celle de Kerenveyer : toutes les deux accordent une grande importance à l'élocution et à la théorie des figures. Toute idée se doit d'être liée à une figure particulière dans le but d'instruire, de plaire ou d'émouvoir - c'est-à-dire de séduire sous les traits d'une figure touchante ou piquante ${ }^{18}$. À lire les figures qu'il met en œuvre dans ses sonnets, ce sont bien là les objectifs de Kerenveyer: ses figures ne sont pas que de simples figures rhétoriques choisies par l'auteur pour se faire valoir ; piquantes, elles

15. Voir François de DAInVILLE, L'éducation des jésuites (XVIe-XVIII siècles), 'le sens commun', les éditions de Minuit, Paris, 1978.

16. Voir Yves LE BERRE, Entre le riche et le pauvre. La littérature du breton entre 1450 et 1650, Brest, Emgleo Breiz, 2012.

17. Voir M.-A. BERNIER, op. cit., p. 114.

18. Voir M.-A. BERNIER, idem, p. 105-106. 
sont destinées à instruire, plaire ou émouvoir - c'est-à-dire à séduire - et elles sont, en ce sens, les fruits bretons des amours cachées de l'enseignement classique, jésuite notamment, et de la pratique littéraire libertine.

Car au service de quoi sont les figures dont j'ai fait le relevé ? Cette amplification caractéristique est au service de quelle pensée ? Dans le premier sonnet, je lis une parodie de l'épisode du livre de Daniel qui met en présence Suzanne et les vieillards ${ }^{19}$. Mac'harit, c'est Suzanne qui se baigne, qui fait sa mijaurée lorsqu'elle est surprise mais qui n'est pas aussi chaste que le laisse entendre l'Ancien Testament. Dans le deuxième sonnet, Kerenveyer rencontre l'amour mais cet amour le quitte, cet amour meurt, le laisse abattu et désireux de mourir - on est subitement passé de la pornographie la plus crue au sentimentalisme larmoyant, ce qui est caractéristique d'une des formes d'humour des écrits licencieux ${ }^{20}$. Dans le troisième sonnet, la vie et le vit restent les plus forts et il suffit de payer pour satisfaire son désir : la femelle dont parle Kerenveyer est peut-être une putain mais il n'empêche que c'est elle qui fixe les règles du jeu et du désir. C'est la cousine de Margot la ravaudeuse, l'héroïne d'un célèbre roman éponyme, écrit par Fougeret de Monbron et publié à Hambourg en $1750^{21}$ : Mac'harit fait montre d'une aussi belle éloquence que celle du Je qui la désire ardemment. Dans ses trois sonnets où est mis en lumière un art de la gradation ${ }^{22}$, Kerenveyer procède bel et bien à une remise en cause des préjugés religieux et sociaux de son temps; les trois figures finales sont piquantes mais elles ne sont pas du seul ressort de l'élocution car elles servent chacune un argument qui en devient plus convaincant parce qu'inventif : les figures de Kerenveyer

19. Voir Historia Susannae / Suzanne et le jugement de Daniel, dans le livre de Daniel (XIII, 1-64).

20. Voir Jean-Christophe ABRAmOVICI, «Les frontières du licite, l'obscénité», dans Histoire de la France littéraire. Classicismes XVIIe - XVIIIe siècle, volume dirigé par Jean-Charles DARMON et Michel DELON, PUF, 2006, p. 435-452, plus particulièrement la p. 446.

21. Publié dans Romanciers libertins du XVIIIe siècle, édition établie sous la direction de Patrick Wald LASOWSKI, Paris, Gallimard, Bibliothèque de la Pléiade, 2000.

22. Voir Michel DELON, «L'art de la gradation», dans Le savoir-vivre libertin, Hachette Littératures, 2000, p. 81-95. 
laissent donc entendre à qui le veut, au cœur de la pointe, un savoir qui n'est pas vraiment celui professé par les curés de campagne du XVIII ${ }^{\mathrm{e}}$ siècle - le Je des sonnets ne se contente pas d'obéir au désir et à ses fureurs, il les met en scène et en mots ${ }^{23}$.

Instruire, plaire et toucher, tel est aussi l'objectif de cette rhétorique mondaine bretonne, art de bien dire, qui de ce fait, comme sa voisine française, pose le problème des connaissances dont doivent disposer les lecteurs ou auditeurs afin d'entendre cette éloquence mondaine et rhétorique ${ }^{24}$. Car derrière les sonnets de Kerenveyer se dessinent des lecteurs potentiels qui ne sont pas les paysans de Keribilbeus ou bien ceux de Poullfaouig. En effet, ces sonnets relèvent très clairement d'une forme de mondanité car, tout comme d'autres textes bretons mondains parvenus jusqu'à nous, ils forment autant d'éléments de clôture ${ }^{25}$ et de connivence ${ }^{26}$, et ils témoignent d'un ensemble de pratiques et de représentations ${ }^{27}$. Ces sonnets sont clos, dans le sens où ils ne sont pas compréhensibles sans clef : les thèmes abordés sont fort éloignés des préoccupations des paysans et ils adoptent une forme très particulière de breton.

Ces sonnets - et le recueil dans son ensemble - illustrent une éloquence qui réduit en figures les arguments d'une pensée philosophique à laquelle adhère, plus ou moins explicitement, Kerenveyer. Selon le tenants de ce qui n'est pas encore nommé sensualisme, il faut écouter son corps et son esprit car la multitude des sensations et des

23. «Le libertin, c'est celui qui, en obéissant à toutes les fantaisies du désir et à chacune de ses fureurs, peut mais doit aussi en éclairer le moindre mouvement par une représentation lucide et volontairement mise en œuvre. Il y a un ordre strict de la vie libertine : toute représentation doit s'animer aussitôt dans le corps vivant du désir, tout désir doit s'énoncer dans la pure lumière d'un discours représentatif» (Michel FoucAult, Les mots et les choses, p. 222).

24. Voir M.-A. BERNIER, op. cit., p. 114.

25. Voir Roland BARTHES, «La Bruyère», Essais critiques, Seuil, «Points Essais», 1981, p. 234-235.

26. Voir Antoine LiLTI, «Mondanité et Révolution : les hommes de lettres et la sociabilité mondaine à la fin du XVIII e siècle», dans Philippe BouRDIN et Jean-Luc ChAPPEY, Réseaux \& sociabilité littéraire en Révolution, Presses Universitaires Blaise-Pascal, Collection Histoires croisées, 2007, p. 49.

27. Voir Antoine LiLti, Le monde des salons. Sociabilité et mondanité à Paris au XVIII siècle, Fayard, 2005, p. 407. 
idées se combine en nous. Cette philosophie popularisée en France par Condillac est une des sources de la production libertine. Soucieux d'analyser nos connaissances afin de découvrir les éléments simples qui les composent et à partir desquelles elles s'élaborent, Condillac en vient à n'admettre plus que les sensations comme source d'où dérivent toutes les idées mais aussi les jugements et raisonnements : il considère ainsi le moi non plus comme une substance pensante existant en soi, mais comme la succession et la transformation de nos sensations. Sa philosophie se propose donc d'expliquer la genèse de toutes nos idées à partir des sensations. Cependant, l'originalité de Condillac réside dans le rôle fondamental qu'il attribue au langage, non seulement dans l'expression des idées mais dans leur élaboration même. C'est le langage qui sert de fondement et de support à la pensée abstraite et réflexive grâce à l'utilisation de signes - d'où la nécessité d'une «langue bien faite», à l'instar de celle formalisée par la grammaire de Port-Royal ${ }^{28}$. Pour Condillac, les signes du langage sont une institution humaine arbitraire : ils assurent la transition des idées directement issues de la sensation aux idées complexes et abstraites de l'entendement et de l'imagination. Les mots disent des idées préexistantes mais contribuent à former des idées nouvelles : plus nous avons de mots, plus nous pouvons avoir d'idées. Penser, juger, raisonner : ces trois actions se résument en un seul art - l'art de parler ${ }^{29}$.

$$
*
$$

Ce n'est pas uniquement parce qu'il s'écrit comme aucun autre breton que le breton mondain est ce qu'il est - les bout-rimés scatologiques abondent en images et métaphores. Il est ce qu'il est également parce que les figures qui l'émaillent mettent en jeu et en scène un savoir, une philosophie de vie : d'une certaine façon, elles facilitent l'argumentation. La figure n'est plus un écart d'expression

28. Voir ARNAULD et LANCELOT, Grammaire générale et raisonnée (1660), éditions Allia, 1997.

29. Voir Michèle CRAMPE-CASNABET, dans la préface à la réédition de l'Essai sur l'origine des connaissances humaines. Ouvrage où l'on réduit à un seul principe tout ce qui concerne l'entendement humain (1746), éditions Alive, 1998, p. 1516. 
extérieur à la pensée ${ }^{30}$ : au service de l'idée, elle la rend convaincante et séduisante - dans le même temps, la figure n'est plus alors du seul domaine de l'éloquence et de l'énonciation ${ }^{31}$.

À l'instar des auteurs de la littérature religieuse de son temps, le discours de Kerenveyer prétend agir sur autrui. Et il ne fait alors qu'appliquer à la lettre ce qu'écrivait Horace, dans son Art poétique : Scribendi recte sapere est et principium et fons.

La raison, voilà le principe et la source assurée du bien écrire.

30. Voir Olivier ReBoul, Introduction à la rhétorique. Théorie et pratique, Presses Universitaires de France, 1991, p. 74-77 et p. 97.

31. Voir M.-A. BERNIER, op. cit., p. 118. 